\title{
Beam Coupling Impedance Measurements And Simulations Of A Beam Pipe Liner With Pumping Holes Or Slots*
}

\author{
E. Ruiz, L. Walling, Y. Goren, N. Spayd \\ Superconducting Super Collider Laboratory \\ 2550 Beckleymeade Ave., MS-4010, Dallas, TX 75237
}

\begin{abstract}
We report the results of measurements using the wire method and numerical simulations using the code HFSS (High-Frequency Structure Simulator) of the beam coupling impedance of the proposed liner for the collider ring of the SSC. We compare the results of the measurements and simulations to analytical estimates by R. Gluckstern[1] and S. Kurennoy[2], and conclude that the additional coupling impedance introduced by a liner with pumping holes can be acceptable.
\end{abstract}

\section{INTRODUCTION}

To avoid reduction of beam lifetime due to photodesorption and to shield the SSC collider ring bore tube from symchrotron radiation, it is proposed to include a holed liner within the bore tube which intercepts the synchrotron radiation while allowing required vacuum to be achieved. Discontinuities in the liner, such as pumping holes or slots internupt the beam image currents, resulting in beam coupling impedance. In addition, electromagnetic coupling through the holes in the liner allow power to circulate between the liner and bore tube in synchronism with the beam. This TEM wave would couple back into the beam pipe, thereby possibly presenting an unacceptably high impedance. The coupling impedance of pumping holes and slots is estimated analytically, and numerical and measurement results are presented.

\section{ANALYTICAL ESTIMATION}

Analytical determination of the coupling impedance of small apertures in metallic walls has been studied extensively, but only recently has their collective coupling impedance in a beam tube liner been described analytically. The pumping holes or slots in the liner excite electric and magnetic dipoles which scatter energy back into the beam pipe and couple some energy through the aperture into the coaxial region formed between the liner and bore tube. Gluckstern calculates the impedance due to holes in a liner including the effect of wall thickness and the outer coaxial region:

*Operated by the University Research Association, Inc. for the U.S. Department of Energy under contract No. DE-AC35-89ER40486
$Z_{\|}=\frac{j Z_{o} k P}{8 \pi^{2} b^{2}}\left[\Psi_{i n}-\chi_{i n}-\frac{\left(\psi_{o u t}-\chi_{o u t}\right)^{2}}{\psi_{i n}-\chi_{i n}-j 8 \pi b^{2}\left(\frac{\alpha}{k}\right) \operatorname{Lln}\left(\frac{a}{b}\right)}\right]$

and

$\mathrm{Z}_{\perp}=\frac{Z_{\| 1}}{n}\left(\frac{2 R}{b^{2}}\right)$

where

$$
\frac{\alpha}{k} \ln \left(\frac{a}{b}\right)=\frac{1}{4}\left(\frac{\delta_{a}}{a}+\frac{\delta_{b}}{b}\right)
$$

and $\Psi_{\text {out }}, \chi_{\text {out }}, \Psi_{\text {in }}$, and $\chi_{\text {in }}$ are the magnetic susceptibility and electric polarizability for the inside and outside of the liner, $Z_{o}$ is $377 \mathrm{ohms}, k=2 \pi \mathrm{f} / \mathrm{c}, P$ is the number of holes, $b$ is the liner radius, $a$ is the bore tube radius, $\delta_{\mathrm{a}}$ and $\delta_{\mathrm{b}}$ are the skin depth for the bore tube and outer surface of the liner, respectively, $L$ is the average axial hole spacing, and $R$ is the ring radius. For small pumping holes, the contribution to the coupling impedance due to power circulating outside the liner is shown by Gluckstem to be negligible.

\section{SIMULATION AND MEASUREMENT}

First, the triaxial geometry for low-frequency longitudinal impedance was simulated. The beam pipe had a radius of 16.5 $\mathrm{mm}$ and thickness of $1 \mathrm{~mm}$. We found that the outer coax contributed insignificantly to the coupling impedance, so to speed up the simulations the outer coax was eliminated. Since holes this size represent a very small impedance, it was necessary to detect minute phase shifts due to the holes. The simulations were converged until the uncertainty in the phase was much smaller than the phase shift $\left(\theta-\theta_{\circ}\right)$, where $\theta_{o}$, the electrical length of the reference liner, was calculated using $c$ specified to about 5 decimal places. However, there was an error that could not be extracted by further adaptive passes when merely subtracting the length of the reference liner. This phase shift is due to small errors in the mapping of the 3-D tetrahedrons to the 2-D port surfaces. Another order of magnitude in phase accuracy was obtained by modeling the holes and the beam tube as separate elements, meshing and running the problem with both specified as vacuum, then, without changing the mesh, re-specifying the holes as metal and re-running over the frequency range. This was used as the "reference pipe" to normalize the data, and removed the port mapping errors as well as the baseline transmission. 
Table 1: Longitudinal Impedance of Various Hole and Slot Geometries at $5.0 \mathrm{GHz}(\mathrm{b}=16 \mathrm{~mm}$, thickness $=1 \mathrm{~mm})$

\begin{tabular}{|c|c|c|}
\hline $\begin{array}{l}\text { Slot } \\
\text { Shape }\end{array}$ & $\begin{array}{l}\text { Longitudinal } \\
\text { Impedance } \\
\text { per Slot }(\Omega)\end{array}$ & $\begin{array}{l}Z / n \text { scaled } \\
\text { to number } \\
\text { required for } \\
\text { vacuum }(\Omega)\end{array}$ \\
\hline Rectangular $(2 \times 6 \mathrm{~mm})$ & 0.0023 & $4.7 \times 10^{-2}$ \\
\hline Rounded (2x6mm) & 0.0020 & $4.3 \times 10^{-2}$ \\
\hline Rounded $(3.2 \times 31 \mathrm{~mm})$ & 0.0172 & $4.0 \times 10^{-2}$ \\
\hline Elliptical $(2 \times 6 \mathrm{~mm})$ & 0.0012 & $3.0 \times 10^{-2}$ \\
\hline Square Hole $(2 \times 2 \mathrm{~mm})$ & 0.0021 & $14 \times 10^{-2}$ \\
\hline Hole $(\mathrm{dia}=3.0 \mathrm{~mm})$ & 0.0075 & $27 \times 10^{-2}$ \\
\hline Hole $(\mathrm{dia}=2.0 \mathrm{~mm})$ & 0.0012 & $11 \times 10^{-2}$ \\
\hline Hole $(\mathrm{dia}=1.5 \mathrm{~mm})$ & 0.00050 & $9.0 \times 10^{-2}$ \\
\hline Hole $(\mathrm{dia}=1.0 \mathrm{~mm})$ & 0.00015 & $7.5 \times 10^{-2}$ \\
\hline
\end{tabular}

\section{A. Longitudinal Impedance}

Measurements were made of a liner 2 meters in length with 1010 pumping holes or 160 rounded slot using the wire technique[3]. Initially, these were made with a center conductor of diameter $12.7 \mathrm{~mm}$, however, simulation of the measurement using HFSS revealed that a $3.2 \mathrm{~mm}$ diameter center conductor yielded more accurate results. For this reason, the longitudinal inpedance data shown in Figure 1 was made with the $3.2 \mathrm{~mm}$ center conductor. In addition, measurements confirmed that the presence of the outer triaxial region did not significantly affect the observed impedance data so later measurements were made without a bore tube.

Many variations of liner holes were simulated. These included variation in the number of holes axially from 2 to 20 , variation of the number of holes longitudinally from 2 to 20 , and varying the depth of the holcs. These simulations confirmed that for low frequencies, the longitudinal impedance of the holes add, and the impedance is reduced by the wall thickness, as theory predicts. Simulations were also performed with small and large center conductors and compared to measurement results for the same parameters. We found very good agreement between HFSS' simulation of the wire technique and wire measurements. Extensive simulations were also performed for comparison with MAFIA simulations done by Chou and Barts[4].

To reduce the low-frequency beam impedance, short slots are being considered. Simulations were nun to study the influence of the shape of the slot and measurements were performed using the wire technique on $3.2 \times 31 \mathrm{~mm}$ rounded slots. The results of longitudinal impedance measurements and simulation at $5.0 \mathrm{GHz}$ for holes and slots are shown in Table 1. A large savings in low-frequency impedance is achieved by using slots; the high-frequency behavior should be acceptable for short slots.

\section{B. Transverse Impedance}

The transverse impedance was measured using two paral-
Table 2: Transverse Impedance of Various Hole and Slot Geometries ( $b=16 \mathrm{~mm}$, thickness $=1 \mathrm{~mm}$ )

\begin{tabular}{|l|l|l|}
\hline \multicolumn{1}{|c|}{$\begin{array}{c}\text { Slot } \\
\text { Shape }\end{array}$} & $\begin{array}{c}\text { Transverse } \\
\text { Impedance } \\
\text { per Slot }(\Omega / \mathrm{m})\end{array}$ & $\begin{array}{c}\text { Z } \\
\text { tometer scaled } \\
\text { required for } \\
\text { vacuum }\left(\Omega / \mathrm{m}^{2}\right)\end{array}$ \\
\hline Rectangular $(2 \times 6 \mathrm{~mm})$ & 0.21 & 71 \\
\hline Rounded $(2 \times 6 \mathrm{~mm})$ & 0.14 & 50 \\
\hline Rounded $(3.2 \times 31 \mathrm{~mm})$ & 0.88 & 34 \\
\hline Elliptical $(2 \times 6 \mathrm{~mm})$ & 0.14 & 58 \\
\hline Square Hole $(2 \times 2 \mathrm{~mm})$ & 0.17 & 185 \\
\hline Hole (dia=3.0mm) & 0.31 & 186 \\
\hline Hole (dia=2.0mm) & 0.087 & 135 \\
\hline Hole (dia=1.5mm) & 0.037 & 111 \\
\hline Hole (dia=1.0mm) & 0.011 & 92 \\
\hline
\end{tabular}

lel center conductors driven $180^{\circ}$ out of phase relative to each other (odd mode). The spacing between the $3.2 \mathrm{~mm}$-diameter conductors is $16 \mathrm{~mm}$. Holes and slots are distributed in rings with 10 holes or slots azimuthally per ring.

Transverse impedance for various hole and slot geometries was also simulated and compared to calculations by Kurennoy for a zero-thickness liner. Kurennoy predicts a factor of 2 reduction in transverse impedance when there are 3 or more holes in one radial cross-section. We find results half of Kurennoy's predictions, which is consistent when the reduction due to wall thickness is taken into account. Simulation results are shown in Tablc 2 whilc mcasurement results are shown in Figure 2.

\section{LINER LEAKAGE}

Measurements of leakage were made using a triaxial impedance measurement technique[5]. The test liner is 2 meters long, with 1010 holes in the center 1 meter, and 0.5 meters of solid pipe on both ends. The outer bore tube has a radius of $23 \mathrm{~mm}$, while the liner has an inner radius of $16 \mathrm{~mm}$ and is $1 \mathrm{~mm}$ thick. The center conductor diameter was 12.7 $\mathrm{mm}$ for the leakage measurements. To measure leakage, the center conductor is placed on axis within the liner and the transmission through the pumping holes, $\mathrm{S}_{\mathbf{4 1}}$, is measured. A transmission measurement is also made through the coaxial regions formed between the liner and bore tube $\left(\mathrm{S}_{43}\right)$, and between the center conductor and liner $\left(S_{21}\right)$, to correct the effects of hardware mismatches and multiple reflections in the triax on the S-parameters. $S_{41}$ is time-gated, and divided by the square root of gated $S_{43}$ and $S_{21}$. Liners with holes of diameter $1 \mathrm{~mm}, 2 \mathrm{~mm}$, and $3 \mathrm{~mm}$ were measured. Results of leakage measurements and simulation are shown in Figure 3.

To estimate the contribution to the coupling impedance of the TEM wave traveling in the region outside the liner and 'feeding back' on to the beam, a two-step calculation was per- 


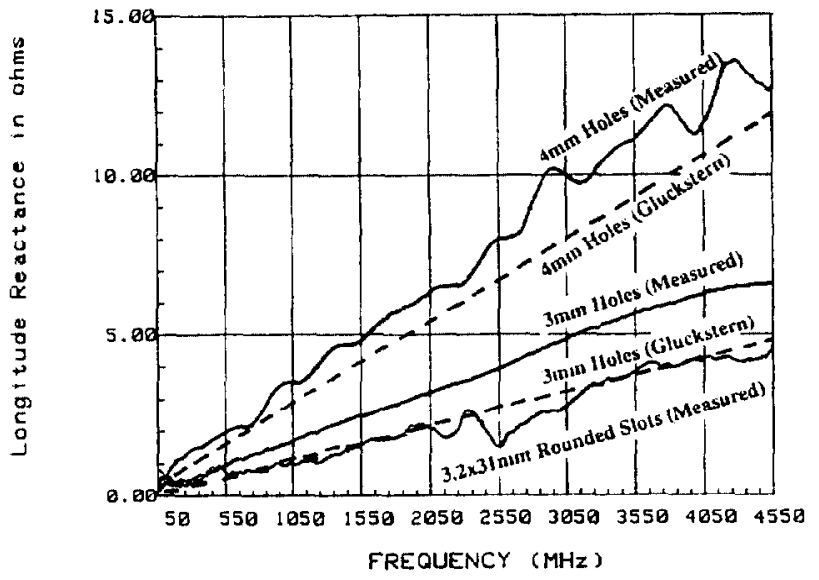

Figure 1. Measured Longitudinal Reactance of $10103 \mathrm{~mm}$ and $4 \mathrm{~mm}$ diameter holes and 160 rounded slots.

formed. A triaxial model representing 20 holes axially and 22 longitudinally, was driven at the inner coax on one side. The other side has enough blark pipe forming the outer coax such that the maximum radial electric field. $\mathrm{E}_{\mathrm{o}}$, due to forwardleaked power could be determined. Thus, the ratio $\mathrm{E}_{\mathrm{o}} / \mathrm{I}$ was determined where $I$ is the current on the center conductor within the liner. Then, the center conductor is removed from the model, the outer coax is driven, and the ratio of $E_{\mathfrak{z}}$, the maximum longitudinal electric field on axis, to the radial electric field in the outer coax is determined. This ratio is then multiplied by the scaling ratio $l_{a t t} / l$ to yield the coupling impedance, where $l$ is the length of the liner segment with holes in it, and $l_{a t t}$ is the attenuation length in the outer coax. Numerical simulations were performed for holes of $2 \mathrm{~mm}$, $3 \mathrm{~mm}$, and $4 \mathrm{~mm}$ diameter. This inquiry confirmed that this contribution was much smaller than the inductive impedance for reasonably-sized holes at low frequencies.

\section{DISCUSSION OF RESULTS}

The simulations and the measurements both yielded lowfrequency longitudinal impedances which are in good agreement with Gluckstern's thickness corrected estimate for the longitudinal impedance of round holes. Transverse impedance for round holes fit Kurennoy's theory well. Slots reduce the impedance but must be kept short to avoid the potentially large coupling impedance caused if the slots themselves become resonant.

We have investigated the high-frequency coupling impedance using $\mathrm{TM}_{01}$ excitation[6]. Because of disagreement between HFSS and MAFIA simulations done by Chou and Barts, we plan to measure the high-frequency impedance[7].

\section{CONCLUSION}

We have performed measurements and simulation of the coupling impedance of a liner in the collider ring of the SSC. Using HFSS we were able to study various hole and slot configurations. Good agreement has been demonstrated between

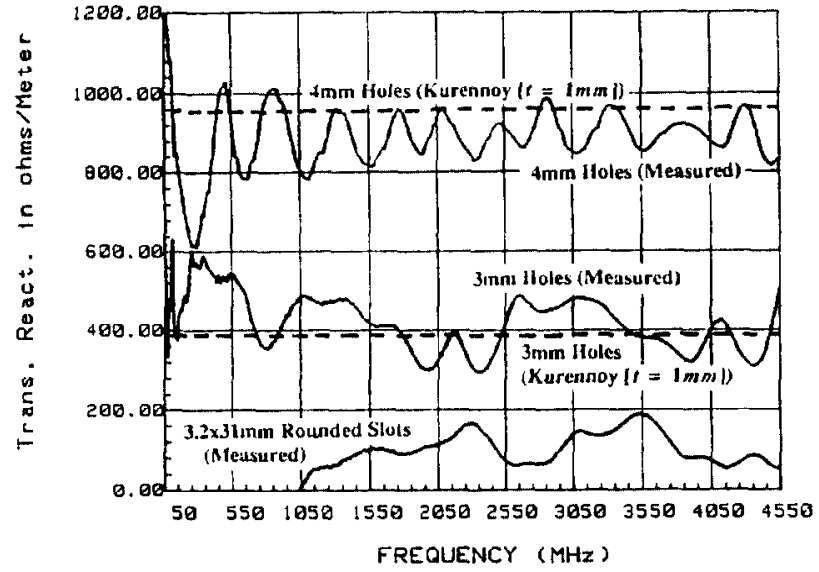

Figure 2. Measured Transverse Reactance of $10103 \mathrm{~mm}$ and $4 \mathrm{~mm}$ diameter holes and 160 rounded slots.

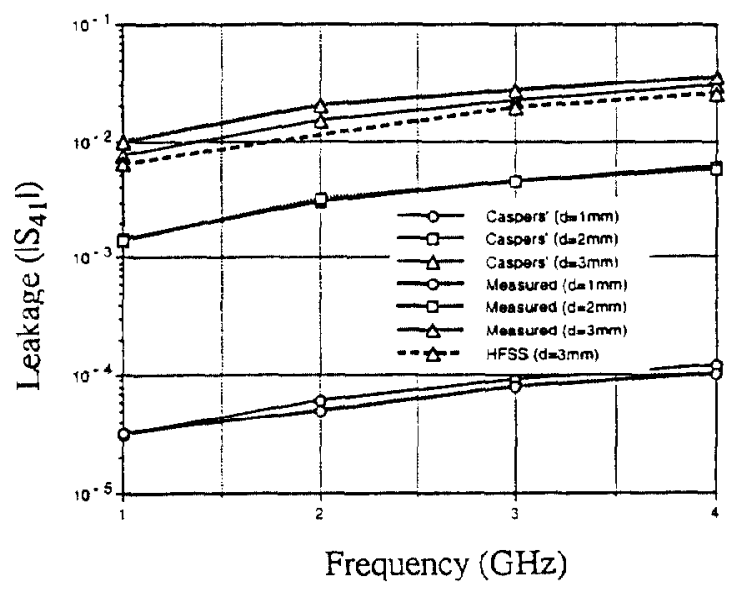

Figure 3. Leakage through a $1 \mathrm{~mm}$ thick liner with 1010 holes of various diameters.

HFSS, laboratory measurements, and theory. Based on these simulations and measurements, the coupling impedance of a liner in the collider ring can be acceptable.

\section{REFERENCES}

[1] R. L. Gluckstern, "Coupling impedance of many holes in a liner within a beam pipe," CERN SL/92-05 (AP) (1992).

[2] S. Kurennoy, "Beam Coupling Impedance of Holes In Vacuum-Chamber Walls," IHEP, Protvino. No.92-84, 1992 [3] "Transmission-Line Impedance Measurements for an Advanced Hadron Facility," L. S. Walling, D.E. McMurry, D.V. Neuffer, and H.A. Thiessen, Nucl. Instr. and Meth. A281 (1989) 433

[4] W. Chou and T. Barts, "Wakefield and Impedance Studies of a Liner Structure Using MAFIA," Proc. of the 1993 Computational Accelerator Physics Conference, Pleasanton, CA. [5] F. Caspers, "Triaxial line technique," in Proc. of the Coupling Impedance Measurement Workshop, Argonne National Laboratory-APS, August 12-13, 1991

[6] E. Ruiz, L. Walling, Y. Goren, "Liner Impedance Calculations Using HFSS," Proc. of the 1993 Computational Accelerator Physics Conference, Pleasanton, CA.

[7] G. Lambertson, A.F. Jacob, R.A. Rimmer, and F. Voelker, "Techniques for Beam Impedance Measurements Above Cutoff," LBL-28190 